Research Article

\title{
A Comparative Study of Aspirin Loaded Bovine Serum Albumin Nanoparticles Prepared by Desolvation Technique Using Various Desolvating Agents
}

\author{
Abbaraju Krishna Sailaja \\ RBVRR Women's College of Pharmacy Affiliated to Osmania University, Hyderabad, India.
}

Corresponding author. E-mail: shailaja1234@rediffmail.com; Tel.: +91-9440182572

Received: Apr. 17, 2017; Accepted: Jun. 2, 2017; Published: Jun. 19, 2017.

Citation: Abbaraju Krishna Sailaja, A Comparative Study of Aspirin Loaded Bovine Serum Albumin Nanoparticles Prepared by Desolvation Technique Using Various Desolvating Agents. Nano Biomed. Eng., 2017, 9(2): |43-I5I.

DOI: $10.5101 /$ nbe.v9i2.p143-151.

\begin{abstract}
Aspirin is mainly used in the treatment of rheumatoid arthtitis and ankylosing spondylitis (AS). The dose of aspirin required for the treatment is $3 \mathrm{~g} /$ day in divided doses. In order to avoid chances of missing the dose of drug, it is better to formulate sustained release dosage forms. In the present study, aspirin loaded bovine serum albumin (BSA) nanoparticles were prepared by desolvation technique using various desolvating agents such as acetone, ethanol and sodium sulphate. A comparative study was made among the three desolvating agents (acetone, ethanol and sodium sulphate) and two methods (continuous addition method and intermittent addition method) to decide the best desolvating agent and the better method for preparation of aspirin nanoparticles by desolvation method. Continuous and intermittent addition methods were followed for the addition of desolvating agent to the aqueous solution of bovine serum albumin. Bovine serum albumin nanoparticles prepared by intermittent addition of ethanol was showing better results with the mean particle diameter of 209 $\mathrm{nm}$, entrapment efficiency of $50 \%$ and loading capacity of $23 \%$. The drug release was slow, extending over a period of $24 \mathrm{~h}$. The curve fitting data revealed that the release followed the first-order kinetics. Higuchis and Peppas plots stated that Fickian diffusion controlled the pattern in all formulations. From the results it can be concluded that the method of intermittent addition was the better method and ethanol was the best desolvating agent for the preparation of aspirin nanoparticles by desolvation technique.
\end{abstract}

Keywords: Desolvation technique; Continuous addition method; Intermittent addition method; Scanning electron microscope; Zeta potential

\section{Introduction}

The major goals in designing polymeric nanoparticles as a delivery system is to control particle size, surface properties and release of pharmacologically active agents in order to achieve the site-specific action of the drug at therapeutically optimal rate and dosage regimen [1, 2]. Due to large surface to volume ratio the nano scale structures have unique properties and dissolution behaviors which are expected to avoid the unwanted side effects. Polymeric nanoparticles are prepared by various techniques like amphiphilic cross linking, polymerization methods and polymer precipitation techniques $[5,6]$. The 
choice of a particular approach depends mainly on the physicochemical properties of the polymer and the nature of incorporated active pharmaceutical ingredients (i.e hydrophobicity / hydrophilicity of the drug and its sensitivity to the solvent) [11, 12]. In this study for the preparation of aspirin loaded bovine seum albumin (BSA) nanoparticles, desolvation technique has been selected $[3,4]$. This technique suitable for natural polymer. Desolvation is a thermodynamically driven self-assembly process for polymeric materials to prepare nanoparticles. The addition of desolvating agents like acetone, ethanol and sodium sulphate are mainly used for the preparation. A balance between attractive and repulsive forces must be present for fabrication of the particles of definite size. The polymeric molecules form particles of different sizes depending on the parameters like $\mathrm{pH}$, ionic strength, cross linking agent concentration, agitation speed, and amount of desolvating agent added. This process includes three steps, including protein dissolution, protein aggregation and protein deaggregation. Finally, the particles are cross linked by using glutaraldehyde as cross linking agent $[7,8]$.

BSA was selected as a polymer. BSA is a globular protein $(66000 \mathrm{Da})$ that is mainly used in numerous biochemical applications due to its stability and lack of interference within biological reaction $[9,10]$.

In this work aspirin has been selected for the study. Aspirin is mainly used in the treatment of rheumatoid arthtitis and ankylosing spondylitis. Aspirin is a non steroidalanti inflammatory drug used to decrease stiffness of the spine and other joints. Ankylosing spondylitis is a form of chronic inflammation of the spine and the sacroiliac joints. The sacroiliac joints are located in the low back where the sacrum (the bone directly above the tailbone) meets the iliac bones. Chronic inflammation in these areas causes pain and stiffness in and around the spine. In the treatment of ankylosing spondylitis and arthritis the dose of aspirin required is $3 \mathrm{~g} /$ day in divided doses. As there are more chances of missing the dose of drug it is better to formulate sustained release dosage forms for better administration. In this study attempts have been made to prepare aspirin loaded BSA nanoparticles by desolvation technique [20, 23].

In the treatment of ankylosing spondylitis (AS) and arthritis the dose of aspirin required is $3 \mathrm{~g} /$ day in divided doses. As there are more chances of missing the dose of drug it is better to formulate sustained release dosage forms for better administration. Aspirin acts by inhibiting cyclooxygenase 1 and cyclooxygenase 2 receptors. By inhibiting cyclooxygenase 1 it causes severe Gastro intestinal bleeding and peptic ulcers. By inhibiting cyclooxygenase 2 , it causes severe cardiovascular problems. To reduce the side effects, dose and dosing frequency a study was made to prepare aspirin nanoparticles by desolvation technique using three desolvating agents

\section{Experimental}

BSA nanoparticles were prepared by desolvation technique using acetone, ethanol and sodium sulphate as desolvating agent $[14,16]$. Weighed amount of BSA powder was added to distilled water. Parameters like $\mathrm{pH}$, stirring speed and volume of desolvating agent to be added were optimized. The $\mathrm{pH}$ was optimized at 7. Two methodologies were followed for the addition of desolvating agent to the aqueous solution of BSA. The first one was the continuous addition method. In this method, the desolvating agent was added at the rate of $1 \mathrm{~mL}$ per minute. The second method was the intermittent addition method. Here the desolvating agent was added at the rate of $1 \mathrm{~mL}$ per every $5 \mathrm{~min}$. The addition was continued till the appearance of turbidity. The appearance of turbidity in the solution indicated the end point of the process. The obtained nanoparticles were stabilized by adding $2 \%$ glutaraldehyde as cross linking agent. Complete cross linking of the formed particles was allowed by continuous stirring for the next $12 \mathrm{~h}$. The residual solvent was removed by means of rota evaporator. The obtained free flowing powder was characterized for particle size distribution to ensure that the particles remain within nano size range. A total of six formulations were prepared by continuous and intermittent addition of acetone, ethanol and sodium sulphate as desolvating agents. All these formulations were characterized for mean particle diameter, drug - polymer interactions and stability. Then the nanoparticles were evaluated for drug content, entrapment efficiency, loading capacity and drug release studies [21, 22].

\section{Results and Discussion}

The particle size of the prepared nanoparticle was determined by scanning electron microscopy (SEM). The images revealed that the particles were showing 


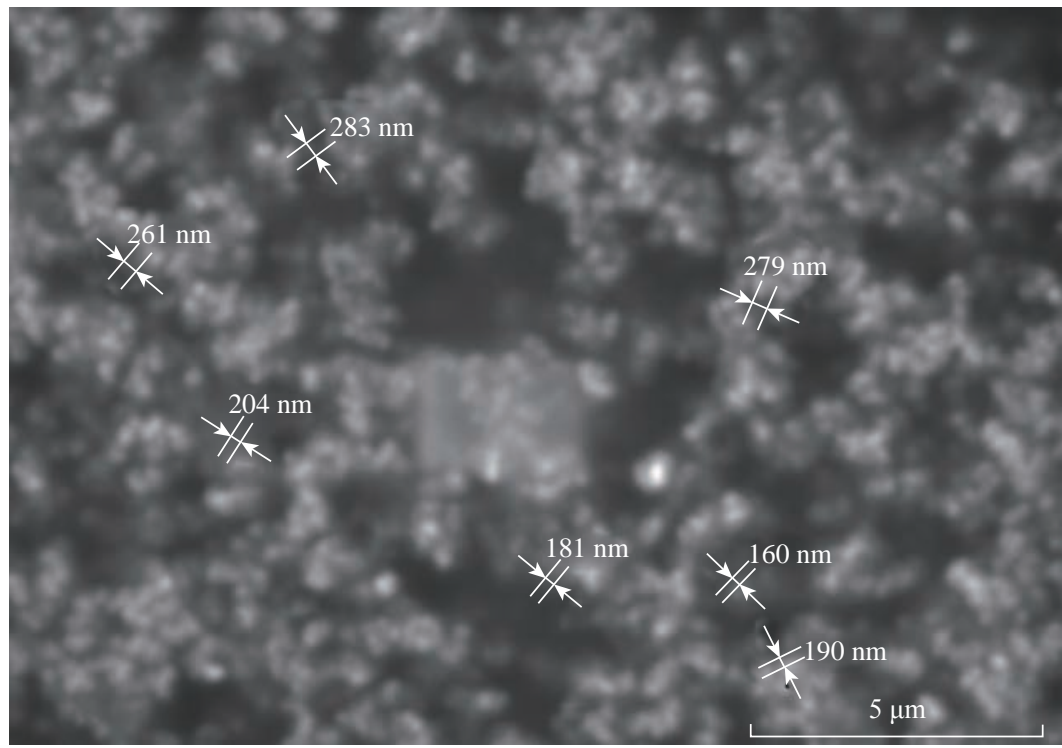

Fig. 1 SEM images of BSA nanoparticles prepared by intermittent addition of ethanol as desolvating agent.

smooth texture and were spherical in shape [18].

\section{Scanning electron microscopy (SEM)}

The sample for the SEM analysis was prepared by sprinkling the nanoparticles on one side of double adhesive stub. The nanoparticles were viewed at an accelerating voltage of $15-20 \mathrm{kV}$ [19]. The particles were found to be in nano range in all the six formulations (Fig. 1).

\section{Particle size analysis}

The mean particle size of the nanoparticles was determined by photon correlation spectroscopy (PCS) with a Malvern Zetasizer Nano ZS (Malvern Instruments, Malvern, UK). Measurements were realized in triplicate at a $90^{\circ}$ angle at $25{ }^{\circ} \mathrm{C}$ under suitable dilution conditions. Particle size distribution was expressed as mean diameter $(\mathrm{nm}) \pm$ standard deviation and polydispersity index. The mean particle diameters of BSA nanoparticles prepared by continuous and Intermittent addition of acetone, Ethanol and sodium sulphate were compared. They were found to be $573.38 \mathrm{~nm}, 321.68 \mathrm{~nm}, 548.2 \mathrm{~nm}$, $209.34 \mathrm{~nm}, 908 \mathrm{~nm}$ and $748.7 \mathrm{~nm}$ respectively (Fig. 2). All formulations were found to be in nano range. When compared, BSA nanoparticles prepared by intermittent addition of ethanol as desolvating agent was resulting particles in nanorange. Probably the intermittent addition gave more time for desolvation process and more stable size was formed. The $\mathrm{pH}$ was the most important factor to control the coagulation of BSA molecules during the desolvation process. The isolectric point (pI) of BSA is about 4.7. When the $\mathrm{pH}$

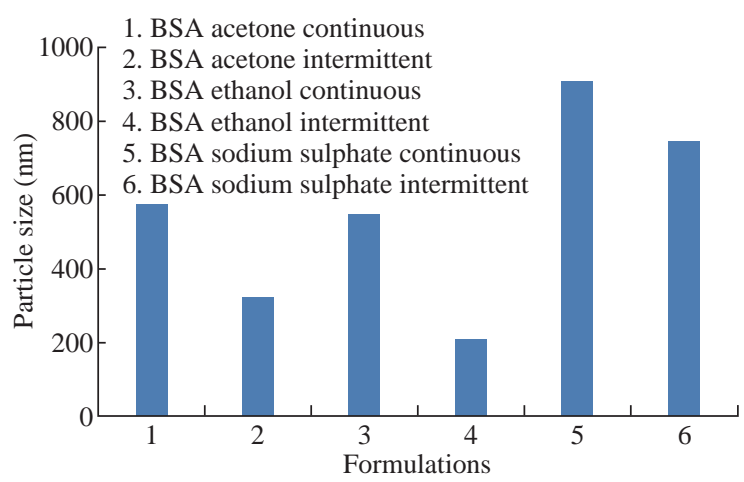

Fig. 2 Comparison of particle sizes of BSA nanoparticles prepared by continuous and intermittent addition of acetone, ethanol and sodium sulphate as desolvating agents.

of solution was closed to the $\mathrm{pI}$, the enhanced proteinprotein reaction lead to increased coagulation among BSA molecules. At pH 7, BSA molecules possessed a negative charge and coagulation of BSA molecules reduced which allowed the formation of small particles.

\section{Zeta potential measurement}

Zeta potential of nanoparticle dispersions was measured in $\mathrm{mV}$ by Malvern Zetasizer Nano ZS (Malvern Instruments, Malvern, UK) in triplicate to determine the surface charge and the potential physical stability of the nanosystem. Zeta potential of nanoparticles was measured in aqueous dispersion. Measurements were realized in triplicate at a $120^{\circ}$ angle at $25{ }^{\circ} \mathrm{C}$. The electrophoretic mobility values of BSA nanoparticles were found to be $-2.436,-2.713$, $-3.123,-4.183,-2.616$ and -3.214 respectively. When compared, the electrophoretic mobility value of 
BSA nanoparticles prepared by intermittent addition of ethanol was higher among all formulations. Zeta potential values of all the six formulations were found to be $-31.1,-34.6,-39.97,-48.7,-33.4$ and -41.39 respectively (Fig. 3). By comparison, zeta potential value of BSA nanoparticles prepared by intermittent addition of ethanol was the highest among all formulations and showed higher stability $[13,15]$.

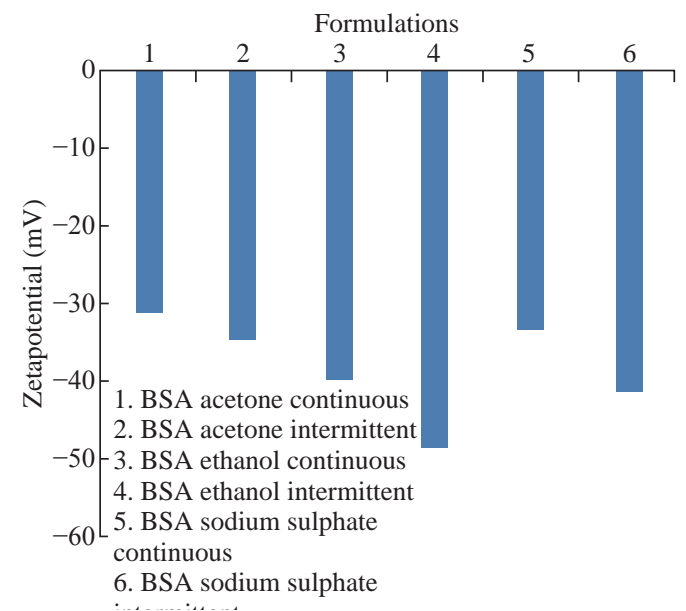

Fig. 3 Comparison of zeta potential values of BSA nanoparticles prepared by continuous and intermittent additions of acetone, ethanol and sodium sulphate as desolvating agents.
Fourier transforms infrared spectroscopy (FTIR)

Compatibility studies were performed using FTIR spectrophotometer. The infrared (IR) spectrum of pure drug and formulations were studied (Fig. 4). The characteristic absorption peaks of aspirin were obtained at wave numbers $2870 / \mathrm{cm}, 2546 / \mathrm{cm}, 1700 / \mathrm{cm}$, $3109.35 / \mathrm{cm}, 1300 / \mathrm{cm}$ and $1085 / \mathrm{cm}$ (K Br disk). The characteristic absorption peaks of BSA were obtained at wave numbers $1641 / \mathrm{cm}, 1680 / \mathrm{cm}, 1696 / \mathrm{cm}, 3000.35 /$ $\mathrm{cm}, 3306 / \mathrm{cm}$ and $2872 / \mathrm{cm}$. The peaks obtained in the spectra of each formulation correlated with the peaks of drug spectrum. This indicates that the drug was compatible with the formulation components.

\section{Percentage yield}

The yields of prepared nanoparticles were calculated. Nanoparticles dried at room temperature were weighed and the yield of nanoparticles was calculated using the following formula:

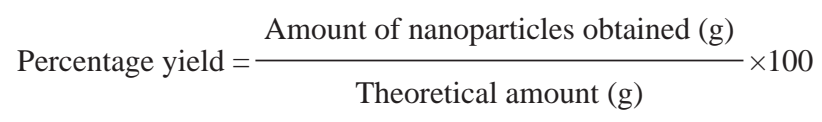

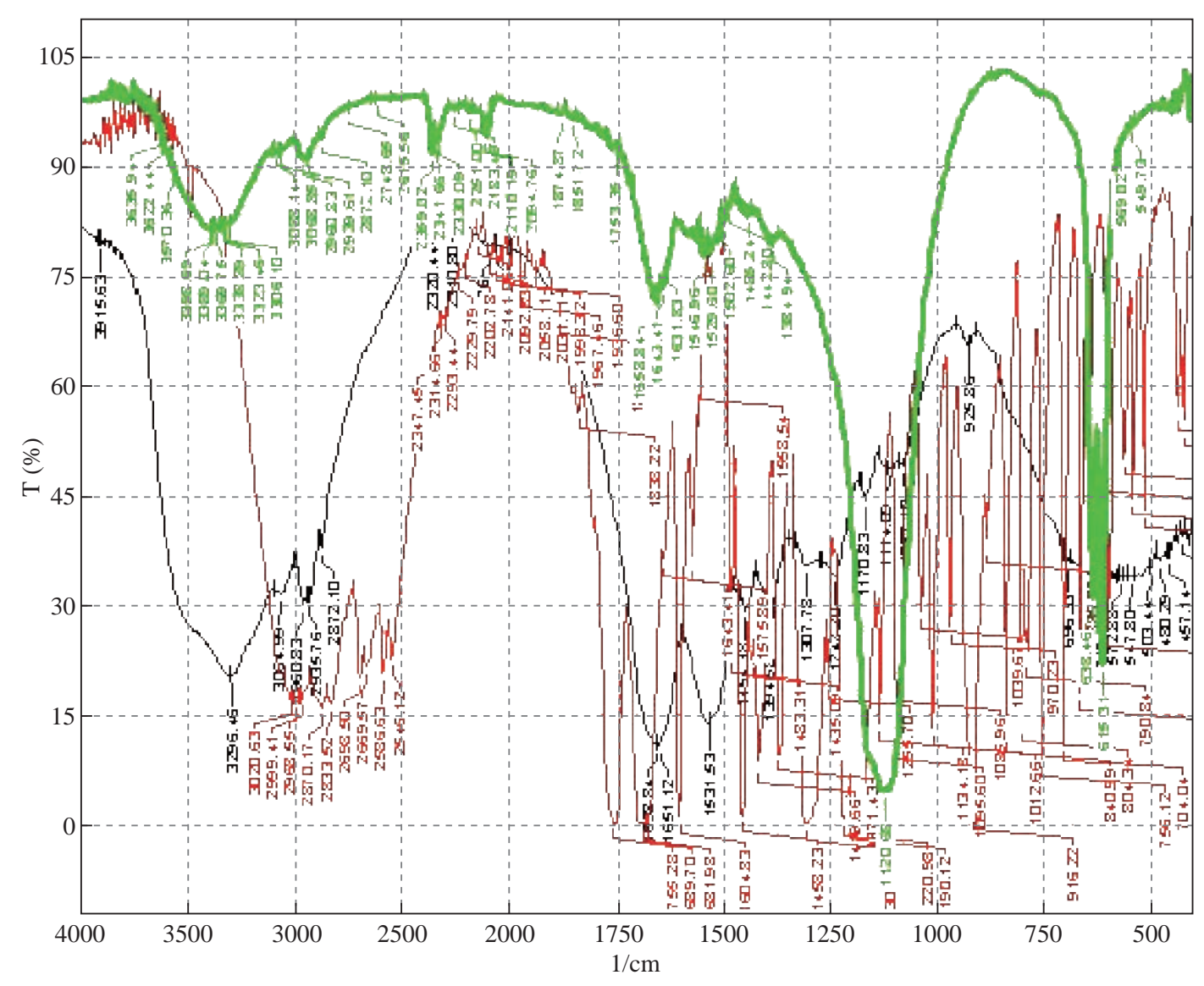

Fig. 4 FTIR spectra of aspirin loaded BSA nanoparticles. 
Product yields of six BSA formulations were compared. They were found to be $75 \%, 91 \%$, $80 \%, 84 \%, 48 \%$ and $60 \%$ respectively (Fig. 5). By comparison, the product yield of BSA nanoparticles prepared by intermittent addition of acetone was the highest among all formulations.

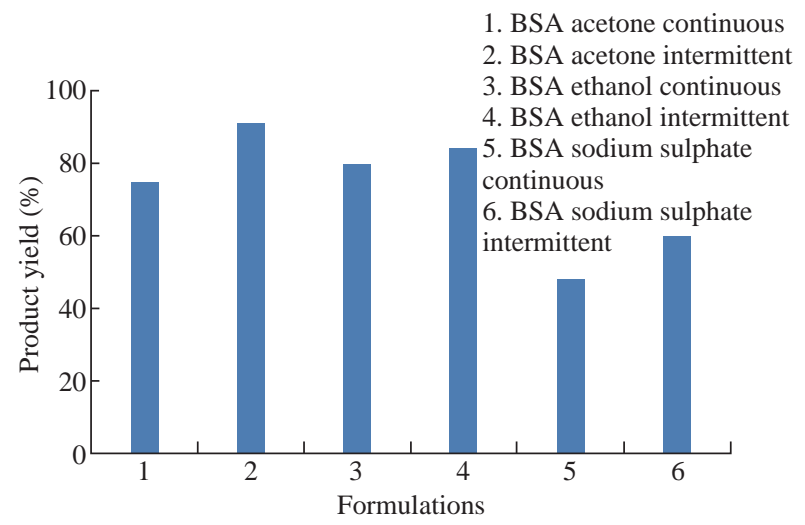

Fig. 5 Comparison of product yields of BSA nanoparticles prepared by continuous and intermittent additions of acetone, ethanol and sodium sulphate as desolvating agents.

\section{Drug content}

Drug loaded nanoparticles were weighed, then grinded to fine powder and dissolved in a solvent in which the drug was completely soluble. It was subjected to stirring around $700 \mathrm{rpm}$ for $3 \mathrm{~h}$. The amount of drug in the supernatant was determined by ultraviolet spectrophotometric method. Drug contents of all the six formulations were compared. They were found to be $89.74 \% 90.5 \% 95.2 \%, 95.7 \% 33.61 \%$ and $32.704 \%$ respectively (Fig. 6). By comparison, the drug content of BSA nanoparticles prepared by intermittent addition of ethanol was the highest among

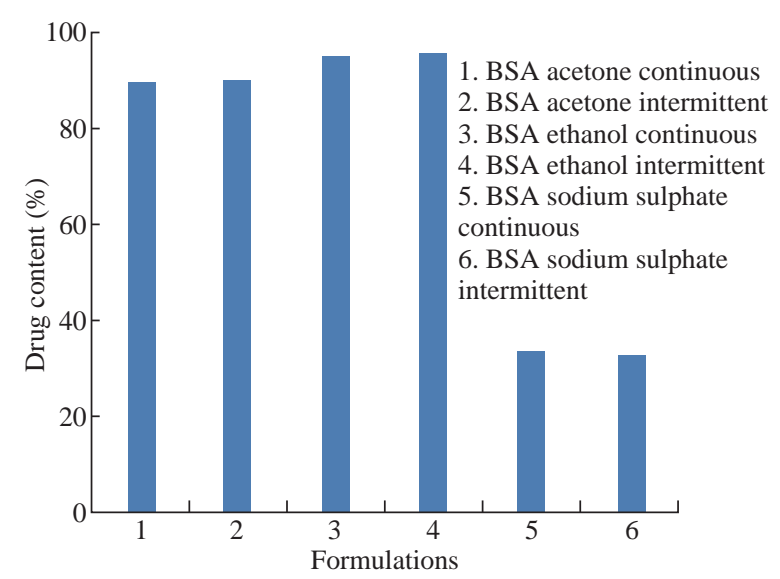

Fig. 6 Comparison of drug contents of BSA nanoparticles prepared by continuous and intermittent additions of acetone, ethanol and sodium sulphate as desolvating agents. all formulations.

\section{Encapsulation efficiency (EE) $[17,18]$}

For the determination of drug entrapment, the amount of drug present in the clear supernatant after centrifugation was determined $(w)$ by ultravioletvisible spectrophotometry (UV-Vis). A standard calibration curve of concentration versus absorbance was plotted for this purpose. The amount of drug in supernatant was then subtracted from the total amount of drug added during the preparation $(W)$. Effectively, $(W-w)$ will give the amount of drug entrapped in the pellet. The percentage entrapment efficiency will be calculated with the following formula:

Entrapment efficiency $=$

$$
\frac{\text { Amout of drug encapsulated in the formulation }}{\text { Total amount of drug in the formulation }} \times 100
$$

\section{Loading capacity}

Loading capacity of a polymer can be determined by the following formula:

L.C. $=$

Total amount of drug - amount of free drug concentration Weight of nanoparticles

Entrapment efficiencies of all six formulations were compared. They were found to be $48.7 \%$, $30.56 \%, 50 \%, 50 \%, 59.25 \%$ and $58.365 \%$ respectively (Fig. 7). By comparison, entrapment efficiency of BSA nanoparticles prepared by continuous addition of sodium sulphate was the highest among all formulations.

Loading capacities were found to be $13.6 \%, 7.64 \%$, $23 \%, 23.2 \%, 12 \%$ and $11.93 \%$ respectively (Fig. 8 ). Loading capacity of BSA nanoparticles prepared by

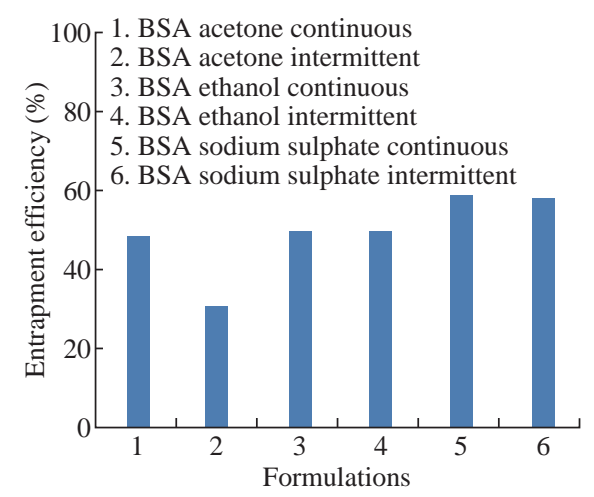

Fig. 7 Comparison of entrapment efficiencies of BSA nanoparticles prepared by continuous and intermittent addition of acetone, ethanol and sodium sulphate as desolvating agents. 


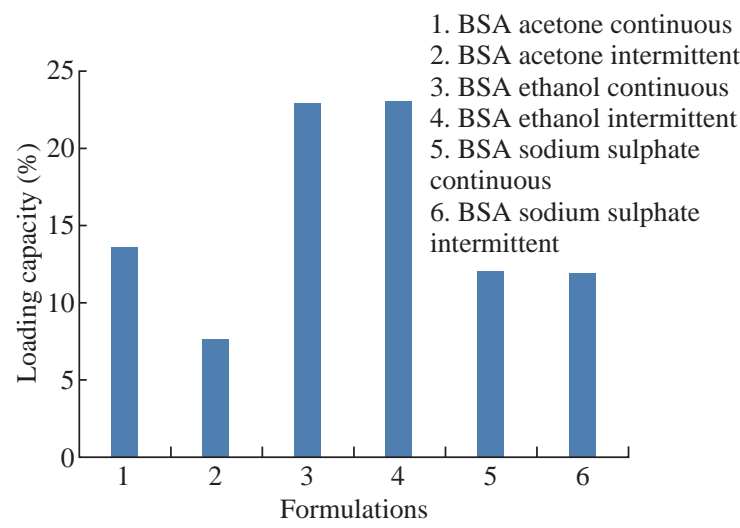

Fig. 8 Comparison of loading capacities of BSA nanoparticles prepared by continuous and intermittent addition of acetone, ethanol and sodium sulphate as desolvating agents.

intermittent addition of ethanol was the highest among all formulations.

\section{Drug release studies}

Drug release studies were performed by means of orbitary shaker. Drug release from polymeric nanoparticles was determined as follows. A known amount of nanoparticles was transferred to a conical flask, and $50 \mathrm{~mL}$ of phosphate buffer with $\mathrm{pH} 7$ was added to the tube. The temperature and rotation were adjusted to $37{ }^{\circ} \mathrm{C}$ and $90 \mathrm{rpm}$, respectively. At the predetermined time of $0.5,2,4,6,8,10,12$, and $24,36,48 \mathrm{~h}, 5 \mathrm{~mL}$ of the sample was removed and ultracentrifuged at $15,000 \times \mathrm{r}$ for $60 \mathrm{~min}$, and $5 \mathrm{~mL}$ of the supernatant was replaced by fresh medium. The samples were further analyzed using UV-Vis spectrophotometer [19].

Invitro drug release of aspirin loaded BSA nanoparticles prepared by continuous and intermittent additions of acetone, ethanol and sodium sulphate as desolvating agents were compared (Fig. 9). They were found to be $98.9 \%, 98.17 \%, 55 \%, 68.09 \%, 31.6 \%$ and $35.32 \%$ respectively. In BSA nano formulations prepared by continuous and intermittent additions of acetone, within a time period of $8.5 \mathrm{~h}, 98.9 \%$ and $98.17 \%$ of drug was released. In BSA nanoparticles prepared by continuous and intermittent additions of ethanol, the drug release was slow, extending over a period of $24 \mathrm{~h}$ and $8.5 \mathrm{~h}$ respectively. With the continuous addition. $55 \%$ of the drug was released in a time period of $24 \mathrm{~h}$. with the intermittent addition, $68.09 \%$ of the drug was released in a time period of $8.5 \mathrm{~h}$. In BSA nanoparticles prepared by continuous and intermittent additions of sodium sulphate as desolvating agent, the drug release was slow, extending

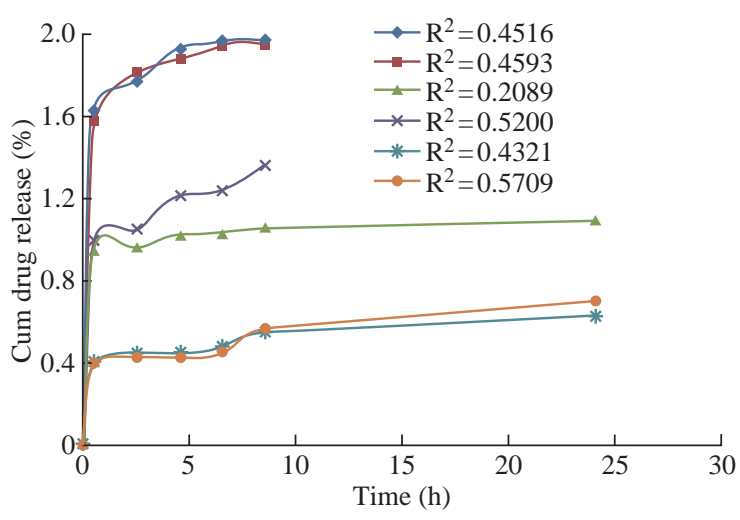

Fig. 9 Comparison of invitro drug release pattern of aspirin loaded BSA nanoparticles prepared by continuous and intermittent additions of acetone, ethanol and sodium sulphate as desolvating agents.

over a period of $24 \mathrm{~h}$. In a time period of $24 \mathrm{~h}, 31.6 \%$ and $35.32 \%$ of the drug was released. Comparatively, continuous and intermittent additions of acetone were resulting in higher drug release rates. The curve fitting data revealed that the release followed the first-order kinetics (Fig. 10). Higuchi and Peppas plots stated that Fickian diffusion controlled the pattern in all the six formulations (Fig. 11 \& 12).

\section{Discussion}

Aspirin loaded BSA nanoparticles were prepared by desolvating technique using acetone, ethanol and sodium sulphate as desolvating agents. Methods of continuous and intermittent additions were followed for the addition of desolvating agent to the aqueous solution of BSA. The $\mathrm{pH}$ was the most important factor to control the coagulation of BSA molecules during the desolvation process. The isoelectric point (pI) of BSA was about 4.7 . When the $\mathrm{pH}$ of the solution was close

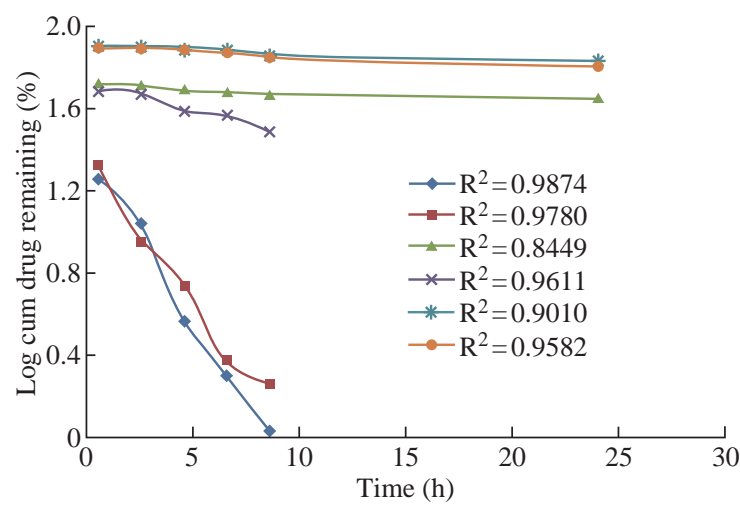

Fig. 10 Comparison of first-order release of aspirin loaded BSA nanoparticles prepared by continuous and intermittent additions of acetone, ethanol and sodium sulphate as desolvating agents. 


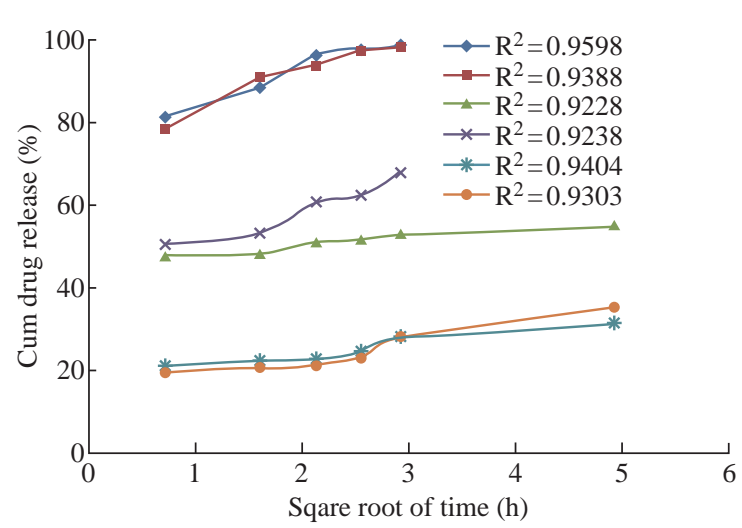

Fig. 11 Comparison of Higuchi square root time dependent plots of aspirin loaded BSA nanoparticles prepared by continuous and intermittent addition of acetone, ethanol and sodium sulphate as desolvating agents.

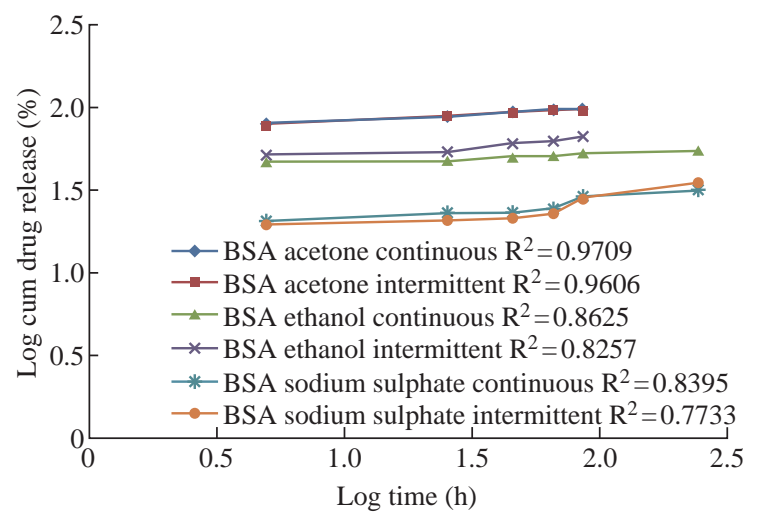

Fig. 12 Comparison of Peppas double log plots of aspirin loaded BSA nanoparticles prepared by continuous and intermittent additions of acetone, ethanol and sodium sulphate as desolvating agents.

to the pI, the enhanced protein-protein reaction led to increased coagulation among BSA molecules. At $\mathrm{pH}$ 7, BSA molecules possessed a negative charge and the coagulation of BSA molecules reduced, which allowed the formation of small particles. By comparison, all formulations were found to be in nano range. When compared, BSA nanoparticles prepared by intermittent addition of ethanol as desolvating agent resulted in particles in nanorange. The intermittent addition probably gave more time for desolvation process and more stable size was formed. Zeta potential was measured by means of Malvern Zetasizer. In a sense, zeta potential represented an index for particle stability. For the case of charged particles, as zeta potential increased, the repulsive interactions would be larger, leading to the formation of more stable particles with a more uniform size distribution. This stability is important in preventing aggregation. By comparison, zeta potential value of BSA nanoparticles prepared by intermittent addition of ethanol was the highest among all formulations and indicated more stability. This could be because of small particle diameter. By comparison, the drug content of BSA nanoparticles prepared by intermittent addition of ethanol was the highest among all formulation. Entrapment efficiency was found to be optimum and loading capacity was the highest among all formulations. Invitro drug release profiles of all six formulations were compared. The drug release was slow, extending over a period of $24 \mathrm{~h}$. In a time period of $8.5 \mathrm{~h}, 98.9 \%, 98.17 \%$ and $68.09 \%$ of drug was released from formulations 1 , 2 and 4 . In formulations 3,5 and 6 , the drug release continued for $24 \mathrm{~h}$ and the drug release was found to be $55 \%, 31.6 \%$ and $35.32 \%$ respectively. When compared, the maximum amount of drug was released from formulations 1 and 2. Among all formulations, BSA nanoparticles prepared by continuous addition of acetone as desolvating agent was showing maximum drug release in a time period of $8.5 \mathrm{~h}$. The curve fitting data revealed that the release followed first-order kinetics; Higuchis and Peppas plots stated that Fickian diffusion controlled the pattern in all formulations.

By comparision, intermittent addition method was considered as the best method for the preparation of nanoparticles, as intermittent addition of acetone, ethanol and sodium sulphate was yielding particles in nanorange when compared to continuous addition. This could be because that intermittent addition gave more time for desolvation process to occur and thus more stable nano particle was formed.

Among the three desolvating agents, ethanol was considered the best desolvating agent because of its stable nanoformulation with a mean particle diameter of $209 \mathrm{~nm}$, good entrapment efficiency of $59.25 \%$ and loading capacity of $23.2 \%$. The nanoformulation obtained by intermittent addition of ethanol was able to sustain the drug release up to $8.5 \mathrm{~h}$ with $68.09 \%$ drug release. The solvents used in this study fell into two categories: polar protic (ethanol and isopropanol) and polar aprotic (acetone). Alcohols are good hydrogen bond donors and acceptors, whereas acetone is not a donor, only an acceptor. This could indicate that the presence of hydrogen bonding favored the formation of larger lattices and consequently of larger nanoparticles [24]. However, the ability of these compounds to interact hydrophobically was also very important. Two parameters were used to compare the polarity of solvents: dielectric constant (D) and polarity index (PI). Acetone being an aprotic solvent, was not able to 
form hydrogen bonds and had a D value of 21 and a PI value of 5.1. Ethanol had an intermediate polarity with a D value of 25 and a PI value of 6.0 (Snyder, 1978). These values suggest that the lower the polarity of the desolvating agents, the larger the hydrophobic interactions and the greater the size of the nanoparticles obtained. This could indicate that the two important characteristics of a desolvating agent to produce large nanoparticles are (1) its ability to form hydrogen bonds and (2) low polarity so it can generate, additionally, hydrophobic interactions. As ethanol is a proteic polar solvent with an ability to act as a hydrogen donor and acceptor, it resulted in better nanoformulation when compared to that of acetone.

\section{Conclusions}

From the results it can be concluded that the best formulation can be obtained by intermittent addition of ethanol as desolvating agent. The drug release continued up to $24 \mathrm{~h}$, indicating its sustained release property. Further research needs to be carried out to design a dosage form for the treatment of rheumatoid arhritis and ankylosing spondylitis after calculating the exact dose to be loaded for single day administration. Invivo studies need to be carried out to conform the data of invitro dissolution studies.

\section{Acknowledgements}

My sincere thanks to the University Grants Commission for providing financial assistance. My sincere thanks to the Principal of College of Technology, Osmania University for providing facilities to carry out the research work.

\section{References}

[1] L.V. Allen, Jr.N.G. Popovich, Ansel's pharmaceutical dosage forms and drug delivery systems. $9^{\text {th }}$ edition. Williams and Wilkins Publications, 2010: 245.

[2] G.S. Banker, N.R. Anderson, L. Lachman, The theory and practice of industrial pharmacy. 3rd edition. Varghese Publication House, 1990: 293-345.

[3] A.C. Tanquary, R.E. Lacey, Controlled release of biologically active agents. Plenum, 1974: 1-13.

[4] S.P. Vyas, R.K. Kha, Controlled drug delivery, Concepts and advances, 1st edition. CBS publications, 2002 1-4: 55-90.

[5] Reilly W.J, Remington. The Science and Practice of Pharmacy, 20th edition, Mack Publishing Company, 2002: 1018-1020.

[6] Brahmankar D.M, Jaiswal S.B, "Biopharmaceutics
\& Pharmaceutics"; First edition; Vallabhprakashan publications, 1995: 335, 162-163, 165

[7] A.F. Hoffman, J.H. Pressman, C.F. Code, et al., Controlled entry of orally administered drugs: Physiological considerations.Drug Dev Ind. Pharm., 1983, 9: 1077-1085.

[8] A. des Rieux, V. Fievez, M. Garinot, et al., Nanoparticles as potential oral delivery systems of proteins and vaccines: A mechanistic approach. Journal of Controlled Release, 2006, 116: 1-27.

[9] M. Rahimnejad, M. Jahanshahi, and G.D. Najafpour, Production of biological nanoparticles from bovine serum albumin for drug delivery. African Journal of Biotechnology, 2006, 5 (20): 1918-1923.

[10] J.Y. Jun, H.H. Nguyen, S.Y.R. Paik, et al., Preparation of Size-controlled Bovine serum albumun(BSA) Nanoparticles by a modified desolvation method. Food Chemistry, 2011, 127(4): 1892-1898.

[11] M. Sivabalan, A. Shering, A. Jose, et al., Formulation and evaluation of 5-Flurouracil loaded chitosan and Eudragit $^{\mathrm{R}}$ nanoparticles for cancer therapy. International journal of Comprehensive Pharmacy. Pharmacie Globale: International Journal of Comprehensive Pharmacy (IJCP), 2011, 2(01): 1-4.

[12] K.S. Soppimath, T.M. Aminabhavi, A.R. Kulkarni, et al., Biodegradable polymeric nanoparticles as drug delivery devices. J. Contr. Release, 2001, 70(1-2): 1-20.

[13] G. Wang, K. Siggers, S. Zhang, et al., Preparation of BMP-2 containing bovine serum albumin (BSA) nanoparticles stabilized by polymer coating. Pharm. Res., 2008, 25(12): 2896-2909.

[14] K. Langer, S. Balthasar, V. Vogel, et al., Optimization of the preparation process for human serum albumin (HSA) Nanoparticles. Int J Pharm., 2003, 257(1-2): 169-180.

[15] R. Mehravar, M. Jahanshahi, and N. Saghatoleslami, Production of biological nanoparticles from lactalbumin for drug delivery and food science application. African Journal of Biotechnology, 2009, 8(24): 6822-6827.

[16] S. Das, R. Banerjee, and J. Bellare, Aspirin loaded albumin nanoparticles by coacervation: implications in drug delivery. Trends Biomater Artif. Organs, 2000, 18 (2): 203-212.

[17] Z.Y. Shen, G.H. Ma, T. Dobashi, et al., Preparation and characterization of thermo-responsive albumin nanospheres. Int J Pharm., 2008, 346(1-2): 133-142.

[18] A.K. Sailaja, P. Amareshwar, and P. Chakravarty, Different techniques used for the preparation of nanoparticles using natural polymers and their applications. International journal of pharmacy and pharmaceutical sciences, 2011, 3(2): 45A-50A.

[19] A.K. Sailaja, P.Amareshwar, Preparation of gelatine nanoparticles by desolvation technique using acetone as desolvating agent. Journal of Pharmacy Research, 2012, 5(4): 1854-1856.

[20] A.K. Krishnasailaja, and P. Amareshwaret al., Preparation of sodium alginate nanoparticles by desolvation technique using iso propyl alcohol as desolvating agent. International Journal of Advances in Pharmaceutics, 2015, 4(5): 60-71.

[21] A.K. Sailaja, P. Amareshwar, et al., Preparation of BSA nanoparticles by desolvation technique using acetone as desolvating agent. International Journal of Pharmaceutical Sciences and Nanotechnology, 2012, 5(1): 1643-1647.

[22] L.R. Snyder, Classification off the solvent properties of 
common liquids. J. Chromatogr. Sci., 1978, 16(6): 223234.

[23] M.A. Ali, M.F. Ali, E. Ciliberto, et al., A new method for the preparation of gelatin nanolayer: A possible approach to the in situ consolidation of damaged gelatinphotographic emulsions. Appl. Phys, 2016, 122552. doi:10.1007/s00339-016-0054-3

[24] A. Taheri, S.M.A. Razavi, Fabrication of cress seed gum nanoparticles, an anionicpolysaccharide, using desolvation technique: An optimization study. Bio Nano Scince, 2015, 5(2): 104-116.

Copyright $(2017$ Abbaraju Krishna Sailaja. This is an openaccess article distributed under the terms of the Creative Commons Attribution License, which permits unrestricted use, distribution, and reproduction in any medium, provided the original author and source are credited. 